\title{
Immunotherapeutic Effects of Dendritic Cells Pulsed with a Coden-optimized HPV 16 E6 and E7 Fusion Gene in Vivo and in Vitro
}

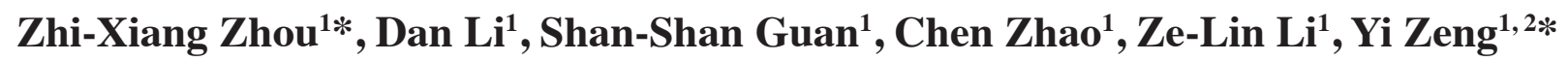

\begin{abstract}
Background: : Cervical cancer is the second most common cause of cancer related death of women. Persistent HPV infection, especially with high-risk types such as HPV16 and HPV18, has been identified to be the primary cause of cervical cancer. E6 and E7 are the major oncoproteins of high-risk HPVs, which are expressed exclusively in HPV infected tissues, and thereby represent ideal therapeutic targets for immunotherapy of cervical cancer. Materials and Methods: In this work, we used recombinant adenovirus expressing coden-optimized HPV16 E6 and E7 fusion protein (Ad-ofE6E7) to prime dendritic cells (DC-ofE6E7), to investigate the ability of primed DC vaccine in eliciting antitumor immunity in vitro and vivo. Results: Our results indicated that DC-ofE6E7 vaccine co-culturing with splenocytes could strongly induce a tumor-specific cytotoxic $T$ lymphocyte (CTL) response and kill the TC-1 cells effectively in vitro. Moreover, DC-ofE6E7 vaccine induced protective immunity against the challenge of TC-1 cancer cells in vivo. Conclusions: The results suggested that the HPV16 ofE6E7 primed DC vaccine has potential application for cervical cancer immunotherapy.
\end{abstract}

Keywords: Human papillomavirus 16 - E6 - E7 - dendritic cells - vaccine - cervical cancer

Asian Pac J Cancer Prev, 16 (9), 3843-3847

\section{Introduction}

Cervical cancer is the second leading cause of cancer death in women worldwide, accounting for $9 \%$ of total new cancer cases and $8 \%$ of total cancer deaths among females in 2008 , and more than $85 \%$ of these cases and deaths occur in developing countries (Jemal et al., 2011). Persistent HPV infection, especially with high-risk types such as HPV16 and HPV18, has been identified to be the primary causes of cervical cancer. The worldwide HPV prevalence in cervical carcinoma is $99.7 \%$, and among them high-risk HPV16 accounts for about 50\% (Aggarwal, 2014). High-risk HPV viral genomes are commonly found integrated into the host cell genome, playing a key role in the process of the formation of cancer (Chansaenroj et al., 2012). The high-risk oncogenic HPVs encode two major transforming genes, E6 and E7, which are required for the immortalization of human primary genital keratinocytes. The high expression of E6 and E7 proteins results in the disruption of cell cycle regulation and leads to genomic instability mainly by promoting degradation of the cellular tumor suppressor proteins p53 and pRb, respectively (Nakagawa et al., 1995; Jiang et al., 2014). Since E6 and E7 are specifically expressed in HPV infected tissues but not in normal tissues, they are ideal molecular targets for the treatment and prevention of cervical cancer.

Traditional treatments of cervical cancer such as surgery, radiotherapy and chemotherapy have their own limitations, and cannot cure cervical cancer. Novel strategies for the treatment of cervical cancer that are effective in reducing the risk of recurrence and metastatic disease are required (Bellone et al., 2007). Dendritic cells (DCs) are the most potent professional antigen processing cells of the immune system with their essential roles in induction and control of T-cell immunity (Kozłowska et al., 2013). Genetic modification of DCs with genes encoding immunoregulatory molecules provides a potential approach for Ag-specific regulation of T cellmediated immunity by selectively targeting antigenspecific T cells (Meixlsperger et al., 2013; Schlitzer et al., 2013). Cellular vaccines based on DCs pulsed with antigens have previously been studied for HPV-associated tumour growth protection or immunotherapy (Ramanathan et al., 2014).

In this study, we used replication-deficient, recombinant adenovirus which expressed coden-optimized HPV16 E6 and E7 fusion-protein to prime dendritic cells, and investigate the ability of primed DC vaccine against TC-1 tumor cells both in vitro and in vivo on C57BL/6 mouse models.

${ }^{1}$ College of Life Science and Bioengineering, Beijing University of Technology, ${ }^{2}$ State Key Laboratory for Infectious Disease Prevention and Control, National Institute for Viral Disease Control and Prevention, Chinese Center for Disease Control and Prevention,Beijing,China*For correspondences: zhouzhixiang@bjut.edu.cn; zengy@public.bta.net.cn 


\section{Materials and Methods}

Mice, tumour cell lines and recombinant adenovirus vector construction

Female 4-6 week old C57BL/6 mice were purchased from Beijing HFK Bio-Technology.co., LTD (China). Animals were housed in Medical Experimental Animal Center of China Academy of Chinese Medical Sciences under pathogen-free conditions.

TC-1 cells are immortalized lung epithelial cells, which was established by transformation of primary C57BL/6 mouse lung cultures with HPV16 E6/E7 oncogenes and activated Ha-ras, was kindly provided by Dr. T. C. Wu of Johns Hopkins University. Cells were cultured in Dulbecco's modified Eagle's medium supplemented with $10 \%$ fetal calf serum, $1 \mathrm{mmol} / \mathrm{L} \mathrm{L}$-glutamine, $100 \mathrm{U} /$ $\mathrm{mL}$ penicillin and $100 \mathrm{mg} / \mathrm{mL}$ streptomycin. Cells were maintained in humidified air containing $5 \% \mathrm{CO}_{2}$ at $37^{\circ} \mathrm{C}$.

The HPV 16 E6E7 fusion gene (HPV 16 ofE6E7) with the termination codon of E6 gene removed was optimized on the basis of the codon usage for mammalian cell expression were described before (Xie et al., 2011). Recombinant adenovirus vector carrying HPV 16 ofE6E7 fusion gene was constructed successfully by our lab.

Adenoviral infection of bone marrow-derived DC to generate the DC-ofE6E7 vaccines

Bone marrow-derived DCs were prepared as described (Lutz et al., 1999). DCs were generated from C57BL/6 mouse bone marrow (BM) progenitor cells as follows: cells from bone marrow were flushed out from hind legs with a syringe, passed through a nylon mesh and collected. Red blood cells were lysed with lysis buffer containing ammonium chloride. Cells were then cultured in RPMI1640 containing 10\% FBS (Life Technologies), rmGM-CSF (50 ng/mL, PeproTech) and rmIL-4 (25 $\mathrm{ng} / \mathrm{mL}$, PeproTech). On the 3th day, half of the culture supernatant was collected and centrifuged and the cell pellet was resuspended in RPMI1640 containing the cytokines. The cells were then returned to the original plate. DCs were matured in the presence of rmTNF-a (100 ng/mL, PeproTech) on the 6th day. Two days later, the DCs were then pulsed with adenovirus at different multiplicity of infection (MOI) for 48h. Percentage and maturation status of the harvested DCs were examined by flow cytometry.

\section{Western blot analysis}

To determine the optimal DC-priming condition, we tested the transduction efficiency by using different MOI during adenovirus infection, which can be monitored by Western blot.

The primed DC were lysed with RIPA lysis buffer (Tris base $50 \mathrm{mM}, \mathrm{NaCl} 150 \mathrm{mM}, \mathrm{NP} 401 \%$, sodium deoxycholate $0.25 \%$, EDTA $1 \mathrm{mM}$ ) containing a protease inhibitor (PMSF). The protein concentration was measured using a BCA protein assay kit (Beijing Zoman Biotechnology Co., Ltd, China). Proteins were separated by SDS-PAGE on $10 \%$ gels and transferred to nitrocellulose membranes (PALL Life Sciences, USA). The membranes were blocked with $5 \%$ non-fat milk in
TBS [Tris-buffered saline ( $\mathrm{pH} 7.4$ )] containing $0.1 \%$ Tween 20 (TBST) at $4{ }^{\circ} \mathrm{C}$ overnight and then incubated with primary antibodies against E6 (1:200 dilution; Santa Cruz, USA) for $2 \mathrm{~h}$. The membranes were then washed four times (5 min each) with TBST and incubated with secondary antibody $(1: 2000)$ for $1 \mathrm{~h}$ at room temperature. Then the membranes were washed four times and the proteins were detected using Odyssey Infrared Imaging System (LI-COR Bioscience, USA).

\section{Detection the specific CTL effect of DC-ofE6E7 vaccine in vitro}

Splenocytes were harvested from female C57BL/6 mice and lymphocytes (effector cells) were enriched by Mouse percoll (DAKEWE Biotech CO., LTD, China). The DC-ofE6E7 cells were co-cultured with lymphocytes for 2 days at the ratio of 1:20 to induce specific CTL. DC cells infected with recombinant adenoviruses expressing empty vector were used as a DC control group.

The effector cells were divided into three groups: DC-ofE6E7 group, DC control group and TC-1 group (TC-1 without CTL as blank control group). After coculturing the effector cells with TC-1 cells (target cells) in 96 well plates at a ratio of 10:1 (effecting cell/target cell, $\mathrm{E} / \mathrm{T}$ ) at $37^{\circ} \mathrm{C}, 5 \% \mathrm{CO}_{2}$ for 2 days. $20 \mu \mathrm{L} \mathrm{CCK}-8$ solution (DOJINDO, Japan) were added into each well. After a further $1 \mathrm{~h}$ incubation, the absorption values at $450 \mathrm{~nm}$ were measured on a microplate reader (Bio-Rad model 550, Richmond, CA, USA). The survival rate of TC-1 cells was calculated according to the following formula: [1- (experimental group-blank control) / (control groupblank control) $] \times 100 \%$.

The effector cells were divided into three groups: DC-ofE6E7 group, DC control group and TC-1 group. After co-culturing the effecting cell with TC-1 cells (target cells) in 6 well plates at a ratio of 10:1 (effecting cell/target cell, E/T ) at $37^{\circ} \mathrm{C}, 5 \% \mathrm{CO}_{2}$ for 2 days. TC-1 cells were then collected by trypsin and washed with cold phosphate-buffered saline. Apoptosis was quantified with Annexin V-FITC / PI Apoptosis Assays Kit (Beijing Zoman Biotechnology Co., Ltd, China) by flow cytometry. The cells at the early stages of apoptosis were annexin $\mathrm{V}$-positive and PI-negative, whereas those at the late stages of apoptosis were both annexin V- and PI-positive.

\section{Detection of the immunogenicity of DC-ofE6E7 cell vaccine ex vivo}

Female C57BL/6 mice were randomly divided into two groups with five mice in each group. One group was immunized with $100 \mu \mathrm{L}\left(1 \times 10^{6}\right.$ cells $)$ DC-ofE6E7 cells suspension by intramuscular injection in the right hind flank. These animals were then boosted three more times using the same regimen at weekly intervals. The remaining group was injected with phosphate-buffered saline (PBS) as control group. Two weeks after the last inoculation with DCs, splenocytes were harvested from the immunized mice. Cellular immune responses were detected using Mouse IFN- $\gamma$ precoated ELISPOT kit (DAKEWE Biotech CO., LTD, China). In brief, splenocytes were plated as $2 \times 10^{5}$ cells in $100 \mathrm{uL}$ RPMI1640 culture medium per well into 96-well plates in triplicates. Samples were 
stimulated with peptides: E7 (49-57) $(4 \mu \mathrm{M})$, E6 (48-57) $(4 \mu \mathrm{M})$, E6 (130-137) $(4 \mu \mathrm{M})$, E7 (49-57) $(4 \mu \mathrm{M})$, E7 (1120) $(4 \mu \mathrm{M})$, the mixture of the above peptides $(4 \mu \mathrm{M})$ and PHA (positive control) and RPMI1640 culture medium (negative control). Then cells were incubated overnight at $37^{\circ} \mathrm{C}$. The plate was washed five times before the addition of the diluted detection antibody (Biotinylated antibody), incubated for $1 \mathrm{~h}$ at $37^{\circ} \mathrm{C}$, and washed another five times with washing buffer. Then the diluted Streptavidin-AP was added and incubated at room temperature for $1 \mathrm{~h}$. After washing, AEC solution was added to each well and the plate was kept away from light for about $15 \mathrm{~min}$ at room temperature. Spots were counted by ImmunoScan (Celluar Technology Ltd, USA).

Detection of immune protection of DC-ofE6E7 vaccine against the challenge of TC-1 cancer cells in vivo

Female C57BL/6 mice were randomly divided into two groups with five mice in each group. One group was immunized with $1 \times 10^{6}$ DC-ofE6E7 cells by intramuscular injection in the right hind flank. These animals were then boosted three more times using the same regimen at weekly intervals. The remaining group was injected with PBS as control group. 7 days after the last inoculation, all mice were inoculated subcutaneously in the right flank with $100 \mu \mathrm{L}\left(2 \times 10^{5}\right.$ cells $) \mathrm{TC}-1$ cells suspension. Tumor volume was measured by caliper weekly. Tumor volume was calculated by the following formula: tumor volume $\left(\mathrm{mm}^{3}\right)=\left(\right.$ length $\times$ width $\left.{ }^{2}\right) / 2$.

\section{Statistical analysis}

SPSS 17.0 statistical software was used for data processing. Two independent groups were compared using t-test statistics. A value of $P<0.05$ was accepted as the level of significant differences.

\section{Results}

Generation of HPV 16 ofE6E7 pulsed DC vaccine transfected with recombinant adenovirus

Immature DCs were isolated from mouse bone marrow via stimulation with GM-CSF, IL- 4 and TNF- $\alpha$ for 8 days and further pulsed with Ad-ofE6E7 for 2 days. The mature dendritic cells expressed high levels of CD11c, CD80, CD86 and MHCIIwhich were analyzed using flow cytometry (data not shown).

To determine the optimal DC-priming condition of Ad-ofE6E7, Western blot was performed to detect the expression of E6E7 fusion protein after DCs were infected by adenovirus with the conditions of MOI at 50, 100, 200, 300, 500. The target band appeared with

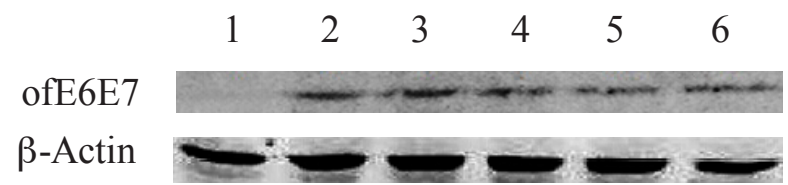

Figure 1. The Optimal DC-priming Condition of AdofE6E7 Detected by Western Blotting. DCs were infected by adenovirus at different MOI. Lane 1, DCs (control); Lane 2, MOI=50; Lane 3, MOI=100; Lane 4, MOI=200; Lane 5, MOI=300; Lane 6, MOI=500 different brightness at different MOI, suggesting that foreign protein E6E7 could be reliably expressed in DCs and $\mathrm{MOI}=100$ was suitable for recombinant adenovirus to infect the BMDCs (Figure 1).

DC-ofE6E7 vaccine inducing specific CTL to decrease the survival rate of TC-1 cells in vitro

The DC-ofE6E7 and DC were co-cultured with lymphocytes for 2 days at the ratio of 1:20 to induce specific CTL. After co-culturing the CTL with TC-1 cells, the survival rate of TC- 1 cells were determined by CCK8 kit. The survival rates of DC-ofE6E7 group, DC control group and TC-1 group (untreated group) were $16 \%, 93 \%$ and $100 \%$ (Figure 2), respectively, which suggests that DCs primed with Ad-ofE6E7 could induce strongly tumor-specific CTL response and kill the TC-1 cells effectively compared to the DC control cells group and the TC-1 cells group.

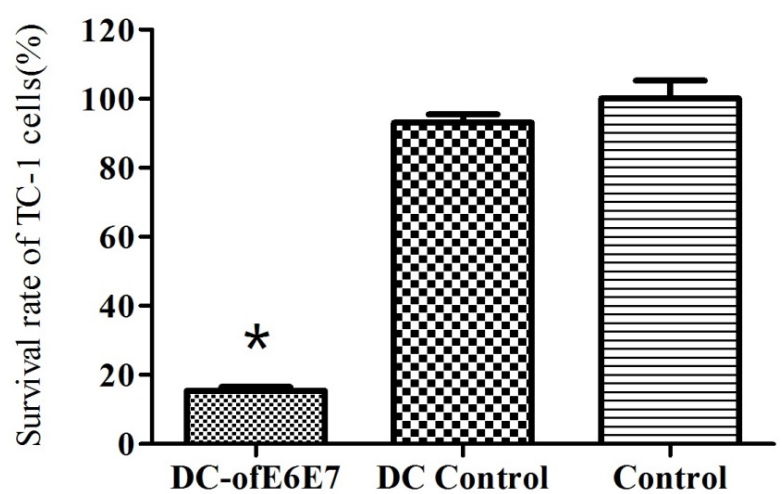

Figure 2. Effect of Specific CTL on the Survival Rate of TC-1 Cells Induced by DC-ofE6E7 Vaccine. TC-1 cells were treated with DC-ofE6E7 vaccine and DC cells. TC-1 cells alone were used as blank control. *Significantly different $(p<0.05)$ when compared to blank control
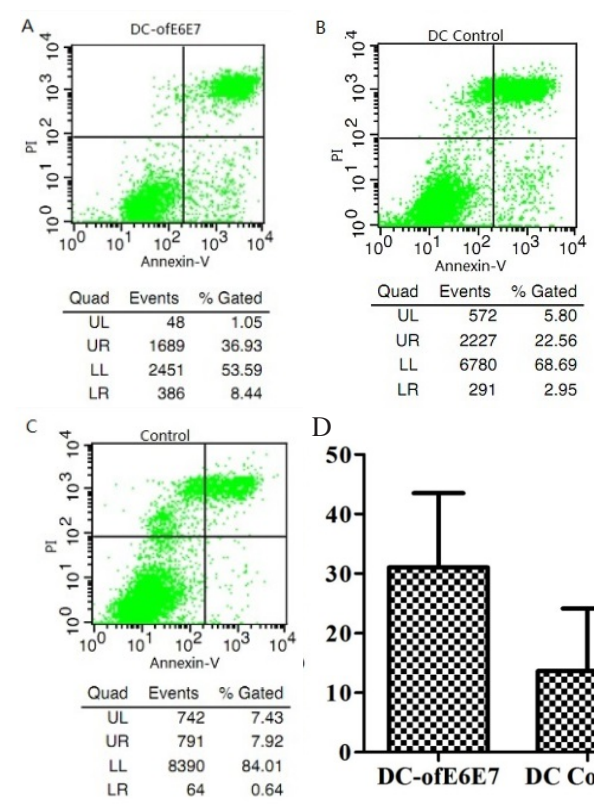

$\mathrm{D}$

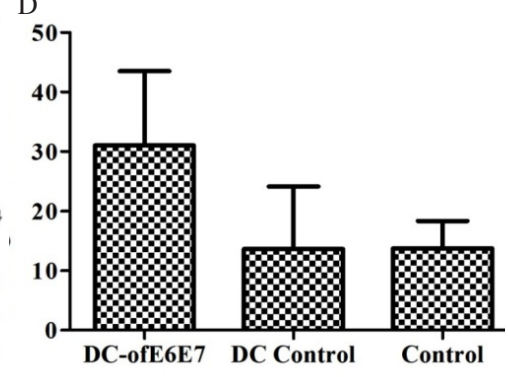

Figure 3. Effect of Specific CTL on the Apoptosis of TC-1 Cells Induced by DC-ofE6E7 Vaccine. (A) TC-1 cells were treated with DC-ofE6E7 vaccine; (B) TC-1cells were treated with DC cells; (C) TC-1 cells alone were used as blank control; (D) The percentages of apoptotic TC-1cells. *Significantly different $(p<0.05)$ when compared to blank control 


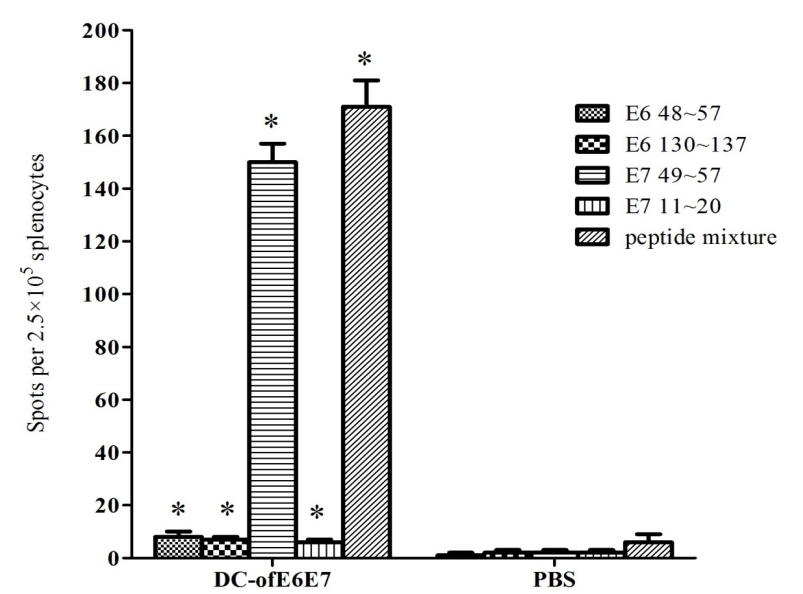

Figure 4. Specific CTL Response Tested Ex Vivo in IFN- $\boldsymbol{\gamma}$ Elispot Assays Against Peptides. Samples were stimulated with $4 \mu \mathrm{M}$ of E7 (49-57), E6 (48-57), E6 (130137), E7 (11-20) and the mixture of the above peptides $(4 \mu \mathrm{M})$, respectively. $*$ Significantly different $(p<0.05)$ when compared to PBS controls

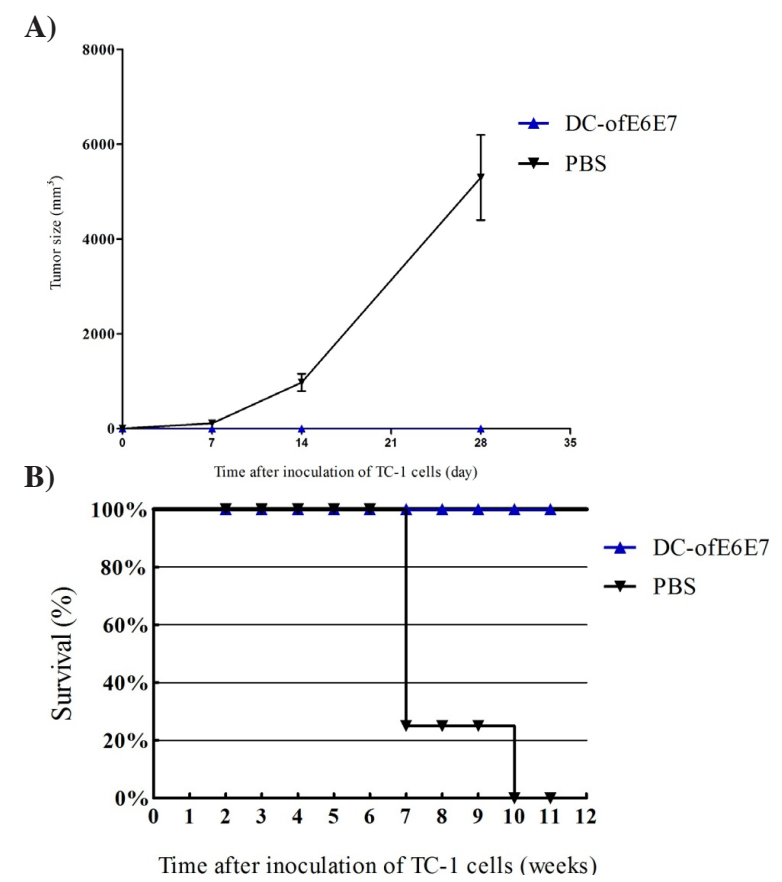

Figure 6. Vaccination with DC-ofE6E7 Induces Protection Against Tumor Challenge. Mice were inoculated with $2 \times 10^{5}$ TC- 1 cells after being immunized four times at weekly interval with DC-ofE6E7 vaccine and PBS. (A) The curve of tumor size after inoculation of TC-1 cells. (B) Survival rate of mice after inoculation of TC-1 cells

DC-ofE6E7 vaccine inducing specific CTL to induce apoptosis of TC-1 cells in vitro

After co-culturing the CTL with TC-1 cells, apoptosis of TC-1 cells was determined by flow cytometry. More than $30 \%$ apoptotic cells were detected in DC-ofE6E7 group, while the percentages of DC control group and the untreated TC-1 cells (blank control groups) were both less than 20\% (Figure 3), suggesting that DCs primed with Ad-ofE6E7 could induce T lymphocytes into specific cytotoxic $\mathrm{T}$ lymphocytes and further eliciting apoptosis of TC-1 cells.
Immunogenicity of the DC-ofE6E7 vaccine ex vivo

To investigate the in vivo immune responses induced by DC-ofE6E7 vaccination, we harvested splenocytes from the immunized mice and further re-stimulated with peptides: E7 (49-57), E6 (48-57), E6 (130-137), E7 (4957), E7 (11-20), and a mixture of the above peptides. Cellular immune responses were examined through the IFN- $\gamma$ producing $\mathrm{CD} 8+\mathrm{T}$ cell populations using an ELISPOT kit. Mice immunized with DC-ofE6E7 vaccines expressed high level of IFN- $\gamma$, compared with the PBS group (Fig.4), suggesting that vaccination with DCofE6E7 cells result in inducing potent cellular immune response ex vivo.

Protection of the DC-ofE6E7 vaccine against tumor challenge of TC-1 cancer cells in vivo

To investigate whether immunizing the mice with DC-ofE6E7 vaccine could provide protection against the outgrowth of the E6- and E7- expressing tumors, mice were inoculated with $2 \times 10^{5} \mathrm{TC}-1$ cells after being immunized four times with DC-ofE6E7 vaccine and PBS. As shown in Figure 5A, mice treated with DCofE6E7 vaccine were completely protected against tumor outgrowth, whereas mice treated with PBS developed large aggressive tumors within 5 weeks, suggesting that prophylactic administration of DC-ofE6E7 vaccines resulted in $100 \%$ protection against the challenge of high dose of HPV E6 and E7-expressing tumor cell TC-1. Accordingly, the survival rates of mice vaccinated with DC-ofE6E7 vaccine were 100\%, whereas mice vaccinated with PBS were all dead after 10 weeks (Figure 5B). The results showed that $\mathrm{DC}$ vaccines prolonged the life-span of immunized mice significantly.

\section{Discussion}

In this study, we verified the usage of DC vaccine primed with HPV16 E6 and E7 fusion protein, and investigated the ability of this DC vaccine in eliciting antitumor immunity in vitro and in vivo.

Human papillomavirus (HPV) is a major etiological factor in cervical cancer, and about $99.7 \%$ of cervical cancers contain HPV, of which $50 \%$ are associated with HPV16 or HPV18 (Jemal et al., 2011). E6 and E7 are the major oncoproteins of high-risk HPVs (Nakagawa et al., 1995; Zhou et al., 2014). Since E6 and E7 are consistently expressed in most cervical cancers and their precursor lesions but do not exist in normal tissues, these oncoproteins are targets for therapeutic vaccines that are presently in development, administering E6/E7 either in live vectors, as peptides, protein or nucleic acid form, or in cell-based vaccines (Seo et al., 2009; Yan et al., 2009; Wu et al., 2014).

Therapeutic vaccines mainly work by eliciting cellular immunity which enabled antigen-specific $\mathrm{T}$ cell into cytotoxic $\mathrm{T}$ lymphocytes to kill target cells. DCs are a crucial part of the vaccine through their ability to capture, process and present antigens to Tlymphocytes (Mellman et al., 2001). Activated DCs which are modified by genes coding for specific tumor antigens enable the priming of CD4+T (Th1) cells and naive CD8+ T cells, and 
elicit effective antigen-specific immunity and antitumor responses (Seo et al., 2009; Yan et al., 2009; Tanchot et al., 2013). In the vaccine production process, most preclinical DC vaccine researchers either pulse the ex vivo cultured DCs with HPV antigen or transfect HPV antigen-coding DNA, RNA or viral vector into the cultured cells (Bellone et al., 2007). These strategies have been shown to be able to induce specific CTL immune responses in mice, and regression of pre-established tumors was also observed in some cases. Various routes of DC vaccine administration have also been studied in preclinical models (Tran et al., 2014).

It has been reported that a recombinant E6/E7 fusion protein is significantly more efficient in inducing antitumor protection than immunization with E6 or E7 oncoprotein alone (Ohlschlager et al., 2009). In addition, studies have demonstrated that E7 specific CTL induction and anti-tumor activity were enhanced significantly through codon modification (Palucka et al., 2012). Therefore, in our study, we used HPV16 ofE6E7, an HPV16 E6 and E7 fusion gene in which the coding sequences were optimized to improve the expression of HPV16 E6E7 in mammalian cells, and pulsed the dendritic cells to generate the DCofE6E7 vaccine for immunotherapy of cervical cancer.

Our results indicate that DC cells primed with AdofE6E7 co-culturing with splenocytes could induce strongly tumor-specific CTL response which could kill or induce apoptosis of the TC-1 cells effectively in vitro. In addition, the detection of IFN- $\gamma$ secreted by effector $\mathrm{T}$ cells explains that a significant cellular immune response was triggered by the primed DC vaccine. Moreover, our DC vaccine induced strong protective immunity against challenge with TC-1 cancer cells in vivo. On the basis of these findings, we suggest that a DC vaccine pulsed with recombinant adenovirus vectors expressing ofE6E7 protein could be developed as an immunotherapy for the treatment of related cancers.

\section{Acknowledgements}

We wish to thank National High Technology Research and Development Program of China (863 Program, No. 2012AA02404/08) and The Key Foundation of the Key State Laboratory (2011SKLID103).

\section{References}

Aggarwal P (2014). Cervical cancer: can it be prevented? World J Clin Oncol, 5, 775-80.

Bellone S, Pecorelli S, Cannon MJ, et al (2007). Advances in dendritic cell-based therapeutic vaccines for cervical cancer. Expert Rev Anticancer Ther, 7, 1473-86.

Chansaenroj J, Theamboonlers A, et al (2012). Whole genome analysis of human papillomavirus type 16 multiple infection in cervical cancer patients. Asian Pac J Cancer Prev, 13, 599-606.

Jemal A, Bray F, Center MM, et al (2011). Global cancer statistics. CA Cancer J Clin, 61, 69-90.

Jiang P, Yue Y (2014). Human papillomavirus oncoproteins and apoptosis (Review). Exp Ther Med, 7, 3-7.

Kozłowska A, Mackiewicz J, Mackiewicz A (2013). Therapeutic gene modified cell based cancer vaccines. Gene, 525, 200-7.
Lutz MB, Kukutsch N, Ogilvie ALJ, et al (1999). An advanced culture method for generating large quantities of highly pure Dendritic cells from mouse Bone Marrow. J Immunol Methods, 223, 77-92.

Meixlsperger S, Leung CS, Ramer PC, et al (2013). CD141+ dendritic cells produce prominent amounts of IFN- $\alpha$ after dsRNA recognition and can be targeted via DEC-205 in humanized mice. Blood, 121, 5034-44.

Mellman I, Steinman RM (2001). Dendritic cells: specialized and regulated antigen processing machines. Cell, 106, 255-8.

Nakagawa S, Wantanabe S, Yoshikawa H, et al (1995). Mutational analysis of human papillomavirus type 16 E6 protein: transforming function for human cells and degradation of P53 in vitro. Virology, 212, 535-42.

Ohlschlager P, Quetting M, Alvarez G, et al (2009). Enhancement of immunogenicity of a therapeutic cervical cancer DNAbased vaccine by co-application of sequenceoptimized genetic adjuvants. Int J Cancer, 125, 189-98.

Palucka K, and Banchereau J (2012). Cancer immunotherapy via dendritic cells. Nat Rev Cancer, 12, 265-77.

Ramanathan P, Ganeshrajah S, Raghanvan R (2014). Development and clinical evaluation of dendritic cell vaccines for HPV related cervical cancer-a feasibility study. Asian Pac J Cancer Prev, 15, 5909-16.

Schlitzer A, McGovern N, Teo P, et al (2013). IRF4 Transcription factor-dependent CD11b (+) Dendritic cells in Human and mouse control Mucosal IL-17 Cytokine responses. Immunity, 38, 970-83.

Seo SH, Jin HT, Park SH, et al (2009). Optimal induction of Hpv DNA vaccine-induced CD8+ T cell responses and therapeutic antitumor effect by antigen engineering and electroporation. Vaccine, 27, 5906-12.

Tanchot C, Terme M, Pere H, Tran, T, et al (2013). Tumorinfiltrating regulatory $\mathrm{T}$ cells: phenotype, role, mechanism of expansion in situ and clinical significance. Cancer Microenviron, 6, 147-57.

Tran N, Hung C, Roden R, Wu T (2014). Control of HPV infection and related cancer through vaccination. Recent Results Cancer Res, 193, 149-71.

Wu X, Liu X, Jiao Q (2014). Cytotoxic T Lymphocytes elicited by dendritic cell-targeted delivery of human papillomavirus type-16 E6/E7 fusion gene exert lethal effects on CaSki cells. Asian Pac J Cancer Prev, 15, 2447-51.

Xie Q, Zhou Z, Li Z, Zeng Y (2011). Transforming activity of a novel mutant of HPV16 E6E7 fusion gene. Virol Sin, 26, 206-13

Yan J, Reichenbach DK, Corbitt N, et al (2009). Induction of antitumor immunity in vivo following delivery of a novel Hpv-16 DNA vaccine encoding an E6/E7 fusion antigen. Vaccine, 27, 431-40.

Zhou Z, Zhao C, Li Q, Zeng Y (2014). A novel mutant of Human Papillomavirus Type 18 E6E7 fusion gene and its transforming activity. Asian Pac J Cancer Prev, 15, 7395-9. 